\title{
McCollough effect: A theory based on the anatomy of the lateral geniculate body
}

\author{
J. KRÜGER \\ Neurologische Universitätsklinik mit Abteilung für Neurophysiologie \\ Hansastrasse 9a, Freiburg, West Germany
}

\begin{abstract}
A model network derived from anatomy and physiology of the monkey retina and lateral geniculate body (LGN) is presented. Control of the strength of concentric surrounds by receptive field center illumination leads to inhomogeneous use of different surround parts, and this occurs even in the presence of irregular eye movements ("autostroboscopic impression"). Various pattern- and movement-contingent color aftereffects, as well as threshold findings, can be explained. Anatomical studies have revealed that the output signals of LGN interneurons are presynaptically controlled at each ending by retinal afferents. This is assumed to result in the above center-surround interaction, and it implies that an interneuron produces different output signals at each of its numerous output endings. The inability of the somatic metabolic machinery to cope with a multitude of different time-varying demands is proposed to be the origin of the remarkably long persistence of the McCollough effect.
\end{abstract}

After a subject has inspected a green-black horizontal and an adjacent red-black vertical grating for some minutes, he will see hues complementary to the stimulating ones when the colors in both gratings are replaced by white (McCollough, 1965). The effect has been studied in great detail by many workers. The subject is extensively reviewed by Stromeyer (1978) and more briefly by Skowbo, Timney, Gentry, and Morant (1975).

Essentially, the effect is monocular, retinotopic, and it may last for hours or even days. The latter remarkable property suggests a relation to memory processes. On the other hand, as monocularity and retinotopy are gradually lost in or beyond the striate cortex, the effect is assumed to occur during relatively early steps of visual processing. Thus, the McCollough effect offers a, to date, unique opportunity to localize and identify a memory process. Many workers, including McCollough (1965), consider the visual cortex as the site of the effect, mainly because of its feature detecting cells, but MacKay and MacKay $(1973,1974)$ suggested that it might occur in the lateral geniculate nucleus (LGN) or the retina by some "cooperative synaptic changes."

In the present work, a neuronal model is presented which describes the results of many psychophysical experiments related to the McCollough effect. The elements of the abstract model are identified with structures and functions of the monkey retina and LGN.

This work was supported by Deutsche Forschungsgemeinschaft (SFB 70, Hirnforschung und Sinnesphysiologie).

\section{Proposed Connection Scheme and Essential Assumptions}

The most common types of retinal ganglion cells (or, briefly, retinal cells) and cells of the LGN parvocellular layers (or, briefly, LGN cells) are considered, namely red on-center/green off-surround and green on-center/red off-surround cells and their off-center/ on-surround counterparts (DeMonasterio \& Gouras, 1975; Gouras, 1968; Krüger, 1977; Wiesel \& Hubel, 1966). The color names stand for cone types. First, the assumed network properties are given, and then they are compared with actual results from the monkey visual system.

Each retinal cell (Figure 1a) has an excitatory connection to a LGN principal cell, or P-cell (large circle), and an interneuron, or I-cell (small circle), which qualitatively have the same receptive field characteristics as their retinal input cells. The P-cells project to the cortex, and the I-cells transmit intrageniculate lateral inhibition. As in the retina, this inhibition is organized in a color-opponent way: red on-center P-cells are inhibited by green on-center I-cells, and vice versa. The lateral inhibition is influenced by a collateral of the excitatory P-cell input (arrows, F). Figure $1 \mathrm{~b}$ shows another aspect of the same network. Lateral inhibition arrives concentrically at a P-cell so that the receptive fields of all cells depicted in the figure are circularly symmetric. In addition, there is a separate, but spatially overlapping, network of off-center cells, where only the + and - signs (for on- and off-functions, respectively) have to be permuted in Figure la but synaptic functions are left unchanged.

An actual connection scheme of the monkey LGN, 
(a)

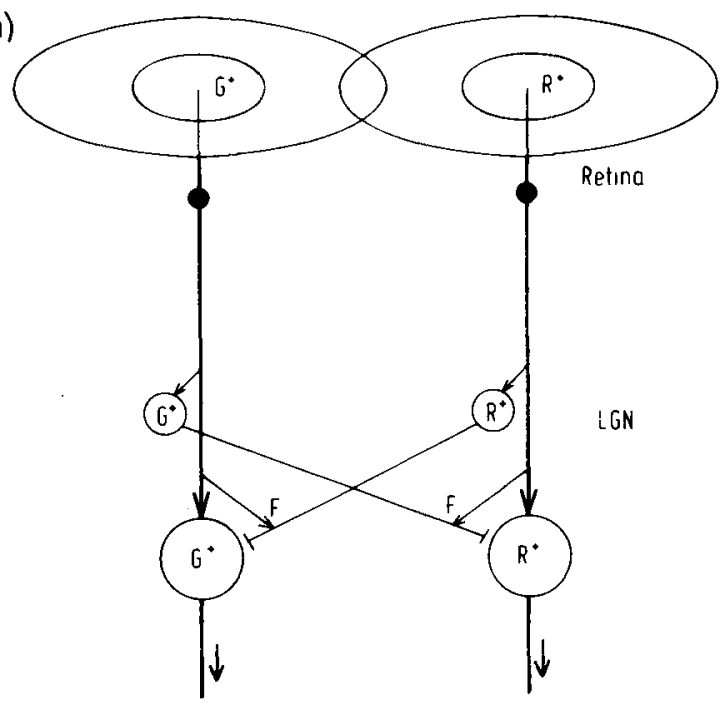

(b)

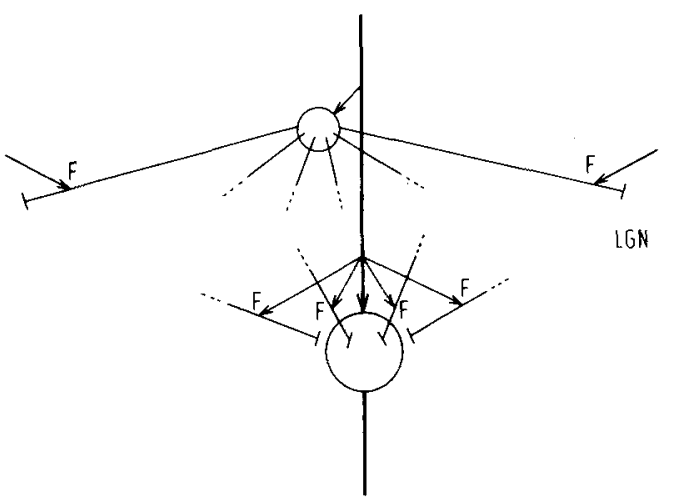

Figure 1. Model network giving rise to the McCollough effect. (a) Two retingl cells (black) each project to a P-cell (large circle) and an I-cell (small circle). The retinal cells have red on-center/ green off-surround (right) or green on-center/red off-surround (left) receptive fields. A P-cell receiving excitation from a red oncenter/green off-surround retinal cell is inhibited by an I-cell whose receptive field is laterally displaced, and whose retinal input has a green on-center. Lateral inhibition of a P-cell is gated (i.e., facilitated in an all-or-none way) at the synapse marked $F$ by the central input. The output of the system is at the bottom. (b) Diagram showing the spatial arrangement within the network depicted in (a). I-cells (small circle) spread their activity in all dịrections in a plane, and P-cells (large circle) receive lateral inhibition from all directions, so that their receptive fields are concentric and grossly similar to those of the retinal cells. Note that the I-cell has many inhibitory outputs which, by virtue of the actions of the F-synapses, can be different at each ending.

as derived from the literature, is shown in Figure 2a. Typically, a retinal terminal is presynaptic to both a $P$-cell and to a dendritic protrusion of an I-cell, which, in turn, is presynaptic to the same P-cell. The optic ending contains round vesicles, the protrusions elongated ones (Colonnier \& Guillery, 1964; Hámori, Pasik, Pasik, \& Szentágothai, 1974; LeVay, 1971; Pecci-Saavedra \& Vaccarezza, 1968; Szentágothai,
1973). Closer to the interneuron's cell body, the morphology of these protrusions resembles that of true dendrites (pr), synaptic vesicles being sparse, whereas more distally (di), vesicles are abundant so that the endings are similar to those of axons (LeVay, 1971).

The juxtaposition in Figure $2 b$ of parts of the anatomical and model networks permits the direct identification of the most crucial elements. The optic afferent

(a)

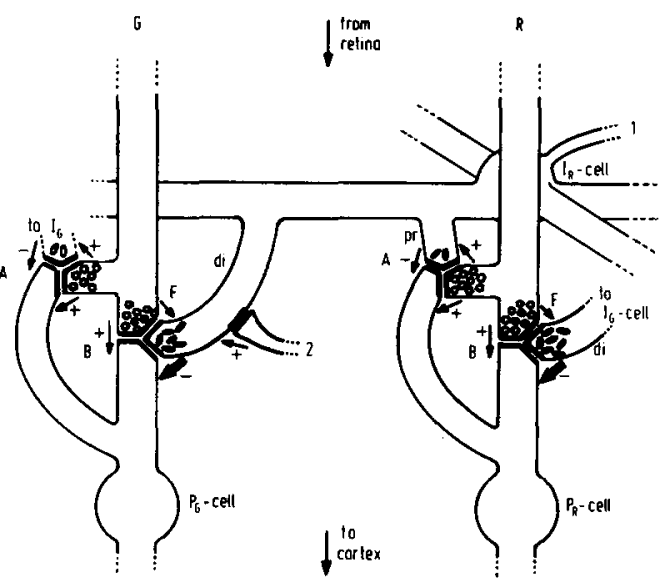

(b)
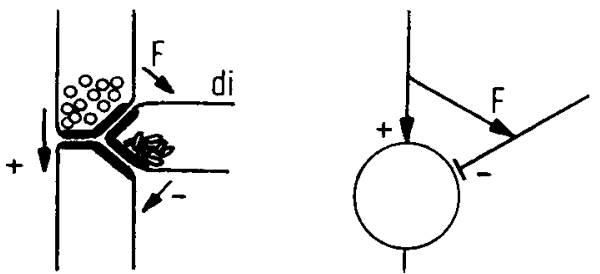

Figure 2. Anatomy of the monkey LGN as derived from works cited in the text. (a) Axons of retinal ganglion cells (from top) form triadic synaptic junctions (at $A$ and $B$ ) directly with LGN $P$-cells (bottom), and with dendritic protrusions of I-cells (upper right), which, in turn, are presynaptic to the same P-cell. Retinal axons contain round, I-cell protrusions contain flattened vesicles. Actually, the latter only face the I-P-synapse. Two types of triads $A$ and $B$ can be distinguished: Those forming contacts with I-cell protrusions proximal (pr) and distal (di) with respect to the I-cell soma. The former contain few vesicles and resemble ordinary dendrites, whereas the latter contain many vesicles so that they appear like axonal endings. This picture derived, from the literature, can be brought into agreement with Figure 1 by the following identifications: Letters and subscripts $R$ and $G$ signify red and green center cone input; plus, minus, and $F$ signs designate excitatory, inhibitory, and facilitatory action, in agreement with vesicle shape and with the arguments presented in the theory. The synapse from I- to $P$-cell at $A$ is considered to have negligible effect, so that this triad can be taken to represent merely two input synapses to the I- and P-cell. The latter can then be unified with the retinal-P-cell connection at $B$. The picture is then congruent with Figure 1, except for the I-cell axon (1), which is not a very prominent structure so that no significance is attributed to it. Corticofugal and nonvisual influences arrive at 2; the excitatory action is only tentative. (b) Identification of the essential elements of the actual anatomy of the monkey LGN (left) and of the model (right). The relations to the whole networks are apparent in Figures 1a, 1b, and 2a. 
has an excitatory $(+)$ connection to the P-cell, and it exerts a control action ( $F$, specified below) upon the distal dendritic protrusion of the I-cell, which transmits lateral inhibition (-).

Further identifications between anatomy and the model are less decisive for the present theory: they are given in the legend of Figure $2 \mathrm{a}$. The validity of the association of excitatory and inhibitory synapses with round and flattened vesicles, respectively, has been reviewed by Gray (1969). Note that the proposed scheme largely agrees with that of Cleland and Dubin (1976) for the cat LGN.

The assumptions which are unique to the present theory are:

(1) The collaterals of the retinal afferents (labelled $F$ in Figure 1) exert an all-or-none facilitatory or gating action on the endings transmitting lateral inhibition. The gate is open (inhibition can pass) when the retinal input governing $F$ is excitatory. This assumption is suggested by results from electrophysiology: According to Wiesel and Hubel (1966), the surround of an LGN cell is difficult to activate if the receptive field center is not illuminated.

(2) (a) High average activity in an intrageniculate lateral pathway leads to a weakening of the efficiency of that pathway ("fatigue"). (b) In any neuron, some metabolites necessary for synaptic function are manufactured by the cell soma, in a quantity proportional to the sum of actual synaptic activity at all output endings. The soma allots the metabolites to the different output endings according to a fixed distribution ratio, irrespective of the differences of metabolite usage resulting from presynaptic facilitation. Depletion of metabolites leads to decreasing synaptic efficiency.

In addition, the following, less unique assumptions are made for retinal cells:

(3) Red light evokes the same response amplitude in red cones as green light in green cones, whereas red in green cones and green in red cones both yield half that amplitude (this follows approximately from Jameson \& Hurvich, 1968). White is taken as red plus green, gray is half as bright as white and appears as bright as red or green. Other colors or intensities are not considered. The choice of the factor $1 / 2$ is not critical.

(4) Responses are sustained, exhibiting no on- or off-transients.

(5) The responsiveness of the total surround is taken to be only $2 / 3$ of that of the center. The center dominance for white homogeneous light is thereby taken into account (Gouras, 1968). The numerical choice is not critical.

(6) Surround responses lag behind center responses.

(7) At each retinal locus, cells with different receptive field center diameters are present. An eccentricity dependence of center sizes is not considered.

\section{Outline of the Model}

In this section, the operation of the model is described in qualitative terms using a special example: consider a subject who inspects ("adapts to") a redblack grating. He is allowed to direct his gaze at any time to any point of the grating so that a succession of different positions of the grating image relative to the retina occurs. In the present example, the adaptation effect on a green on-center/red off-surround $P$-cell is treated whose receptive field center diameter matches one bar width of the grating. The P-cell receives its excitatory input from one retinal cell of the same type as the P-cell itself. The responses of the retinal cell to various stimuli are determined in Section 3, but for the present example it is sufficient to realize that the retinal cell, although it has a receptive field center linked to green cones, is excited by the red grating, but only when a red bar is well centered in the receptive field: The green cones are well stimulated by red light (see Assumption 3), and a maximal portion of the more red-sensitive inhibitory surround is in darkness. However, a fairly small decentration of the red bar already turns the response into an inhibition. The succession of excitation and inhibition which is obtained when all grating positions within one period are tested is depicted as a dotted curve ("retinal response function") in the top middle frame of Figure 3. So far, this is what is expected from known properties of retinal cells. The following step is specific to the present model: The retinal cell not only excites the P-cell to which it projects, but whenever it is excited it allows the same P-cell to be subjected to lateral inhibition (see Assumption 1). The elevated portions of the small rectangular curve ("gating function") in the top middle frame of Figure 3 indicate for which grating positions the gate admitting lateral inhibition is open.

Now consider the grating as it changes its retinal position in some arbitrary way. As soon as a position is reached in the range where the retinal cell is excited, the gate opens and lateral inhibition can act upon the P-cell. The inhibition is strong, since the grating is red and the inhibitory surround is formed by red on-center I-cells (see Section 1). Of course, inhibition comes only from those parts of the surround of the P-cell which are at that instant covered by the red bars. This leads to an inhomogeneous use of the surround: it operates only when one red bar is located in the P-cell's receptive field center. The mechanism is termed "autostroboscopy" because, although the stimulating pattern moves, the surround "sees" it only when it assumes a defined position, as under stroboscopic illumination. "Auto-" indicates that it is the pattern itself which determines the active phases.

Each point of the P-cell's surround is linked to the 
P-cell by an I-cell appendage whose synaptic ending experiences fatigue proportional to use (Assumption $2 a)$. Thereby the grating produces a fatigue replica impressed in the surround ("autostroboscopic impression'). For the present example, the pattern is shown as a perspective drawing at top left in Figure 4. The ensemble of undulated lines represents a surface whose most elevated points correspond to the most fatigued parts of the surround. The way of obtaining such surfaces is described in Section 4 in greater detail, and in Section 5 the results for different patterns are presented.

The reasoning for understanding the psychophysical test which reveals the effect of adaptation is simple: During testing, a P-cell must be excited in order to signal the test pattern to the cortex, but at the same time lateral inhibition selectively elicited in the most fatigued surround parts must operate. This is described in Section 6.

The proposed adaptation mechanism may be further clarified by the consideration of the action of the same red-black grating on a red on-center/green offsurround P-cell. In this case, the grating excites the red on-center retinal afferent for nearly all positions (Figure 3, top center frame, continuous curve), so that no sharply tuned stroboscopic effect can occur. Moreover, as the lateral inhibition in the P-cell is mediated by green on-center I-cells, the surround fatigue is weak. The resulting shallow fatigue pattern is shown in Figure 4 (top, second from left).

No further points are treated in this outline, since in the corresponding sections they are presented in a less formal way.

\section{Responses of Retinal Cells to Gratings: Results of Computations for Various Color and Brightness Contrasts}

First, separate sensitivity profiles for center and surround are chosen (Assumption 5 is incorporated):

$$
\begin{array}{ll}
\text { center: } & \frac{1}{\pi} e^{-r^{2}} \\
\text { surround: } & \frac{1}{3 \pi} r^{4} e^{-r^{2}},
\end{array}
$$

where $r$ is a relative radial coordinate measured from the receptive field center. The center sensitivity falls to $1 / \mathrm{e}$ at $\mathrm{r}=1$, which is the center radius. The surround sensitivity has its maximum (for small spots) at $r=\sqrt{2}$, where it reaches $18 \%$ of the peak center sensitivity. A red homogeneous field evokes a response contribution of unity from the center mechanism of a red on-center cell, and half that value is elicited by a green homogeneous field.

Responses of retinal cells to luminance and color patterns as a function of a retinal position coordinate are obtained by convolution of the light distributions with the above sensitivity profiles. Depending on color, center and surround components are weighed according to Assumption 3, and for an off-center or an off-surround the corresponding sensitivity profile is taken as negative.

First, the responses to gratings for red on-center/ green off-surround and green on-center/red offsurround (referred to as red and green cells) are calculated. Off-center cells are treated later. Alternating bars are equally wide and have infinite length. Bar widths of $1 / 2,1$, and 2 times the receptive field center diameter, and red/black, red/gray, red/white, black/white, and red/green gratings are considered. The convolution calculations are performed for different grating positions along an X-axis perpendicular to the grating bars, with each position corresponding to a different gaze direction of the subject inspecting the pattern. The results ("retinal response functions") are periodic functions of $x$ (Figure 3). The responses are excitatory (inhibitory) for positive (negative) sections of the curves.

"Gating functions" derived from Assumption 1 are depicted in Figure 3 below each pair of retinal response functions. They simply translate positive or negative parts of the response functions into unity or zero ordinate values, respectively.

\section{Intrageniculate Lateral Inhibition and Neuronal Fatigue: Computations for the Case of Unrestricted Gaze Direction}

Response functions for I-cells are identical to those of their retinal afferents. Lateral inhibition transmitted by an I-cell ending is proportional to the excitatory part of the response function of a laterally located I-cell, multiplied by the gating function of the central $P$-cell afferent.

The application of Assumption $2 b$ is postponed to Section 6. Instead, here the simpler Assumption 2a is used, since it yields the same results for most spatial and chromatic aspects. Neuronal fatigue in an intrageniculate lateral pathway is proportional to the average transmitted lateral inhibition. It is obtained by the integration over all gaze directions of the products calculated above. The case of fixation is considered in Section 8.

\section{Adaptation Results for the Case of Unrestricted Gaze Direction}

Integration results reflecting the effects of exposure ("adaptation") to various gratings are shown as perspective drawings in Figure 4. Each series of undulated curves correspond to a two-dimensional surface covering the receptive field surround of a $P$-cell. The height of the surface at a particular surround 


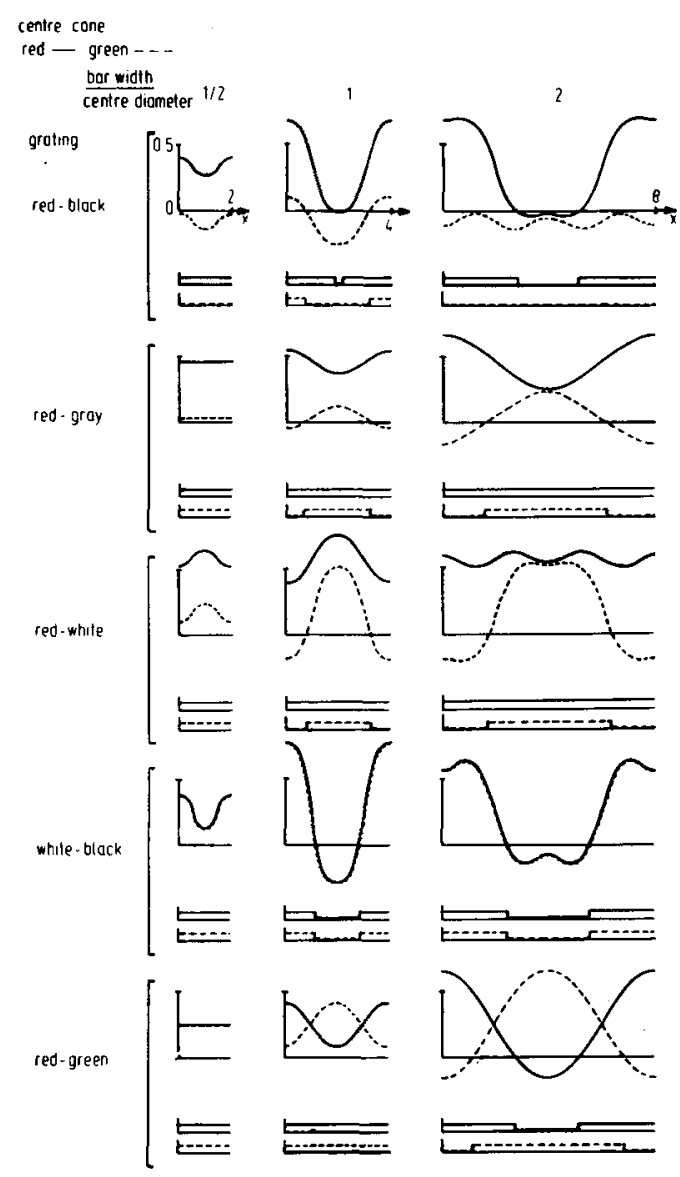

Figure 3. Retinal response functions and gating functions of red and green retinal cells for various gratings. For each grating, characterized by the light distribution (indicated at left) and the bar width relative to the receptive field center diameter (indicated at top), there are four curves: Two retinal response functions (smooth curves), corresponding to the cell's activation as the grating is exposed on the retina at different positions relative to the receptive field center, and two gating functions (small frames), which are unity (zero) for positive (negative) values of the corresponding response functions. All curves also apply when red and green in the grating and dashed and continuous curves are interchanged. Horizontal scales, $x$, are the same for all frames in a column. The unity is the receptive field center radius. At $x=0$, the middle of the colored (for the white-black grating, the white) stripe is in the receptive field center. Each curve is one cycle of a periodic curve, and the cycle corresponds to one pair of grating bars. The ordinate scale valid for all response functions is given at top left, the significance of its unit is given in Section 3 .

locus indicates the neuronal fatigue of a synapse linking the I-cell having its receptive field at that locus to the central P-cell. The small annulus represents diameter and location of the receptive field center of the P-cell. The surround extension is not crucial here: The outer limit is only tentative, and it is not critical whether or not the surround mechanism is actually present in the receptive field center. Note that all fatigue patterns have a fixed position relative to receptive field centers of $\mathbf{P}$-cells, no matter where the centers are located. Thus, in the case of two P-cells with overlapping receptive fields, the same retinal locus may belong to a strongly fatigued surround part of the one cell and to a nonfatigued part in the other.

In the figure, only the nontrivial cases are presented. The largest modulation of neuronal fatigue is evoked by a red-black grating in a green on-center $P$-cell whose receptive field center diameter matches one bar width (top left; according to Assumption 3, this holds also for a green-black grating and a red on-center P-cell).

Gratings composed of red and gray stripes of equal brightness produce the patterns shown in the second from top row of Figure 4: For bars matching the receptive field center diameter, the fatigue modulation
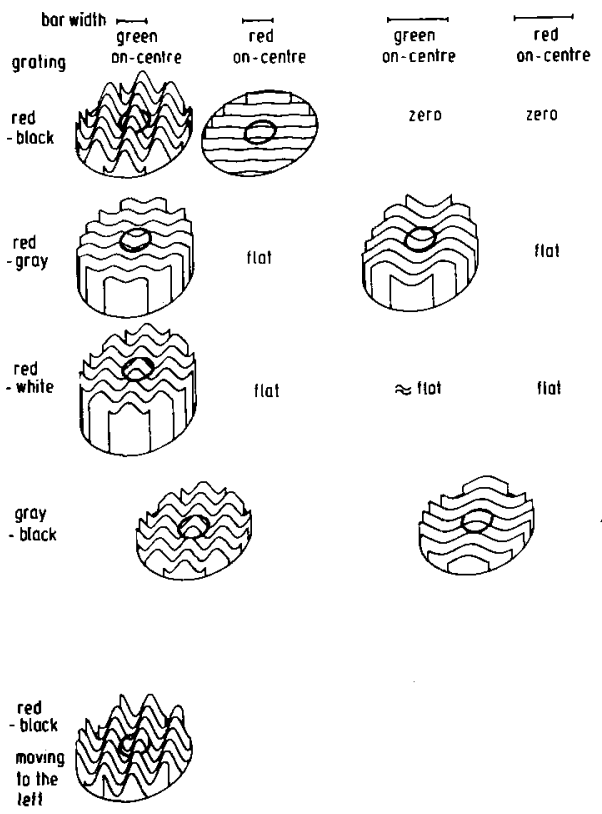

Figure 4. Neuronal fatigue of LGN surround center connections after inspection of various patterns. The perspective drawings show undulated surfaces over the P-cell surrounds. The height of a surface at a particular point of the surround indicates the amount of fatigue present in the inhibitory pathway connecting that point to the $\mathbf{P}$-cell's receptive field center. The diameter of the latter is indicated by an annulus. The trivial flat and zero cases have been either omitted (for bar width/center diameter $=1 / 2$ ) or are indicated by words. For simplicity, all results have béen approximated by sinewaves. Top three rows: Colored gratings viewed with unrestricted gaze direction. It can be seen that gratings with red bars mainly affect green on-center cells. Except for a dc-level, the resulting fatigue patterns are similar for the red-black and red-white gratings, whereas an equiluminant red-gray grating produces a slight reverse effect, which is also apparent for smaller receptive fields (or wider bars). The effect of a gray-black grating on red and green on-center cells is equal and similar to the effects of red-black and red-white gratings on green cells (second from bottom row). Bottom row: Effect of a red-black grating moving to the left, with the gaze direction held stationary. Here, a distanceindependent surround response lagging behind the center response is assumed. 
is weak and the modulation phase is reversed. Again, the grating has no effect in a red on-center cell, but a grating with wider bars evokes fatigue variations in a green on-center cell (second from top, right). The color-white gratings, in turn, with white appearing brighter than the color, have effects very similar to those of color-black gratings, except for a higher dc level (Figure 4, middle row). Gray-black gratings have equal effects in red and green on-center cells (second from bottom row).

A red-black grating drifting to the left evokes an asymmetric pattern, as shown in Figure 4 (bottom): When a red bar reaches the receptive field center of the retinal green on-center cell providing excitatory input to the P-cell, the gate is opened and lateral inhibition generated earlier (Assumption 6) flows in. If, instead of a single delay between center and surround, a conduction time lag increasing with lateral distance is introduced, the crests and valleys of the surfaces become angled, or curved, as shown in Figure 5 (left). It is noteworthy that an identical pattern is generated by stationary curved bars.

A pattern of red dots in a black field elicits lateral inhibition in the surrounds of green on-center/red off-surround cells, but the inhibition is only effective at those instances when eye movements have carried the pattern to a position where one red spot is well centered in a green on-center receptive field of a $\mathbf{P}$-cell, so that a maximum of the most sensitive surround parts adjacent to the center are in darkness. The green cell is then excited and the gate for lateral inhibition is opened. Fatigue appears in the surround

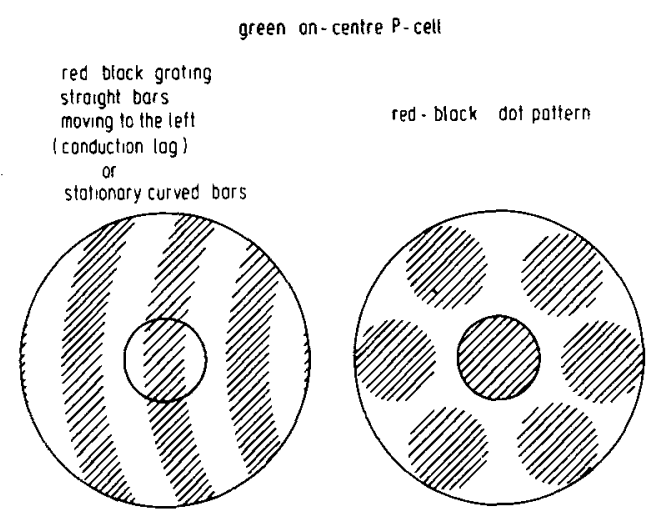

Figure 5. Neuronal fatigue of $L$ GN center-surround connections after inspection of various patterns. Patterns of fatigue distribution analogous to those of Figure 4 are shown as top views here, with the hatched areas representing the most fatigued portions. The patterns are qualitative illustrations only; they are valid for green on-center cells and red-black patterns. Left: Patterns produced either by a stationary grating with convex-left curved bars, or by a left-moving grating with straight bars, when a distancedepending time lag of surround responses is assumed. Right: Pattern resulting from adaptation to a hexagonal array of red dots. parts hit by the dots in these instances (Figure 5, right). Just as in the case of gratings, a green dot pattern can only have weak effects in the same cell.

Red and green homogeneous fields yield no intrageniculate lateral inhibition: Either the central excitatory or the lateral inhibitory input to a P-cell is zero, because one of the retinal on-center cell types is inhibited. White homogeneous fields excite both pathways, but the resulting fatigue is spatially unmodulated, which is a trivial case treated in Section 8.

Modulated fatigue in off-center cells occurs according to the same principles as in on-center cells: It is generated when a stimulus with appropriate modulation excites the central as well as some lateral inputs. Figure 3 can be consulted because offcenter cells are excited when on-center cells of the same color opponent type are inhibited. It can be seen that only white-black gratings and the coarsest red-green grating (which is trivial due to the general red-green symmetry) can elicit significant excitations in red and green off-center cells. The pattern for the white-black grating is similar to that of the on-center cells: It has a crest in the center, but the undulations are shallower.

It remains to be stated that the population of LGN cells may at the same time have different fatigue patterns impressed into the surrounds of different cell types. The most prominent example is a redblack grating having affected green on-center cells, as shown at top left in Figure 4. Subsequent inspection of a green-black grating produces a similar fatigue pattern in red on-center cells, which so far had nearly unaltered surrounds. The case with the red grating rotated by $90^{\circ}$ against the green grating is of particular experimental interest. Similarly, unaffected cells with larger or smaller receptive fields may receive an independent impression of some stimulus whose dimensions are suitably chosen. Finally, a red-black grating moving to the left and a greenblack grating moving to the right, inspected in succession, affect green and red on-center cells, respectively, in a quasi-independent way.

\section{Tests Revealing the Effects of Fatigue}

The message sent from an LGN cell to the cortex will most strongly deviate from standard when a $\mathrm{P}$-cell is exposed to a test pattern which selectively stimulates the most altered intrageniculate lateral pathways, and which at the same time causes a significant firing in the P-cell. The best compromise for both center and surround stimulation is given by a white- (or gray-) black grating. Consider the green on-center cell with the fatigue pattern of Figure 4, top left. The achromatic grating excites the cell most strongly when a white bar is in the receptive field center, and at these moments the surround is most activated in its most weakened parts. Therefore, the cell is less inhibited than usual, and consequently, its response is stronger than in the nonfatigued case. This gives rise to the greenish appearance of the achromatic test grating. The red P-cell, having an unaltered surround, does not contribute a response deviating from standard. 
In general, the strength of a McCollough aftereffect depends on the relationship between the spatial parameters of the test and adaptation patterns, and mostly, for maximal effects, this relationship must simply be a match. This is in contrast to the idea of many workers that essentially the pattern must be matched to stimulus requirements of a specific cell. In the present theory, only one parameter, namely the pattern scale factor, is linked to the receptive field center size.

Accordingly, adaptation to a red-black dot pattern (Figure 5, right) yields a greenish hue in a whiteblack dot pattern of the same dimensions, and similarly, the optimal color aftereffect for a moving adaptation grating is obtained for the same spatial frequency and velocity of the test grating, revealing the direction selectivity resulting from adaptation.

A white-black adapting grating produces equal adaptation aftereffects in red and green on-center P-cells, and it is the only nontrivial stimulus which is able to produce significant fatigue in off-center cells. Therefore, the present theory predicts an increase in apparent contrast when test and adapting grating are identical.

\section{Long Persistence of the McCollough Effect}

In the previous section, neuronal fatigue occurring in pathways transmitting intrageniculate lateral inhibition was treated. In this section, in addition, the mechanism of metabolite supply providing relief of fatigue is considered.

The "classical" neuron may be defined as a cell which receives a multitude of inputs via dendrites but which emits a single output signal via a possibly branching axon. The activities at all axonal endings are always proportional to each other, and so are their demands of metabolites. It seems sufficient, then, that the cell soma produces the metabolites at a well-matched activity-dependent rate and allots them according to a fixed distribution ratio to the individual endings. (Metabolites provided by other sources are disregarded.) Retinal ganglion cells and LGN P-cells are examples of "classical" neurons in the network considered.

The essence of Assumption $2 b$ is that all neurons have identical systems of metabolite supply, namely that of the "classical" neuron. Even the I-cell is included, which does not conform to this picture: The individual actions of the facilitatory (or gating) synapses (see Figure lb) have the effect that each ending produces a different output signal so that individual amounts of metabolites would have to be provided to the endings. Instead, according to Assumption 2b, a fixed share of the metabolites manufactured by the soma is allotted to each ending, and the manufacture rate is adjusted to the average consumption of all endings. Under natural viewing conditions, an excess of metabolites delivered at one instant to an ending which was less active than the average will be compensated statistically at other instants when other stimuli evoke more-than-average activity in the same ending. A difficulty arises when one set of endings is prolongedly more active than another, and no compensatory stimuli are provided. This is the case when the stimuli considered in Section 4 are inspected. The best thing the metabolic machineries of the I-cells can do is to relieve the average fatigue of all endings taken together. This amounts to lowering the dc-level of the undulated surfaces in Figure 4 to zero. Clearly, this does not remove the undulations, and if no further stimulus is applied, they remain indefinitely. This explanation of the long persistence of the McCollough effect differs from the more common fatigue explanation implied by Assumption 2a alone insofar as the effect is viewed as a residue of inadequately relieved fatigue.

"Negative fatigue," or excess of metabolite supply, is locally created when the dc-level is lowered as described above. The question of an accompanying increase in synaptic potency is considered in Section 10.

\section{Adaptation to Gratings Under Steady Fixation}

For the explanation of results obtained under steady fixation, it is more important to take Assumption $2 b$ into account than for the condition of unrestricted eye movements.

The first case to be considered is a simple one: Gating functions which are constantly zero or unity have the effect that all output signals of a given I-cell are identical (zero included) so that the I-cell functions like a "classical" neuron (see previous section). The metabolic machinery can cope with this situation so that no long-lasting aftereffects remain. The simplest example for this case is a homogeneous white field. Other cases are red-green (in particular, fixated) gratings for bar widths equal to or smaller than the center diameters of $\mathbf{P}$-cells: The flat gating functions are shown in Figure 3. Red-green gratings with larger bar widths do show fatigue patterns for appropriately located P-cells: The intrageniculate surrounds become weakened in directions perpendicular to the bars, whereas in parallel directions they remain unaffected. (A detailed demonstration of this result is omitted for brevity.) A black-white fixated test grating, wherever it is located, mainly tests the surrounds in the latter directions, and therefore, for this case too, no aftereffect is expected.

At the input level to green P-cells in the LGN, red-black, red-gray, and red-white gratings all evoke spatially modulated gating functions for a bar width/ center size ratio of unity. For an explanation of corresponding aftereffects, an alternative interpretation of Figure 3 is convenient: The response and gating 
functions should be viewed as evoked by a fixed grating in a sheet of cells. Thus, for a given grating, there is one "retinal response surface" fixed on the retina and there are fixed "gate-open" and "gateclosed" stripes where the former surface is positive or negative, respectively. Fatigue can only develop in the surrounds of those P-cells whose receptive field centers are located in the "gate-open" stripes, because in the "gate-closed" stripes the inhibitory I-cell endings are not active. As can be seen in Figure 3, the "gate-open" stripes move from the red to the white bar positions as black is gradually changed to white: In the top middle frame, the gate (dotted rectangle) is open around $x=0$, whereas in the third from top middle frame this is at $x=2$. As a result, similar fatigue patterns develop under the red stripes of a red-black grating and under the white stripes of a red-white grating. As for the case of unrestricted scanning, no large aftereffect is expected for the redgray grating.

\section{Thresholds}

According to the present theory, the prolonged inspection of a red grating leaves red on-center $P$-cells unaffected but lateral inhibition is weakened in green on-center P-cells. Near threshold, the latter mediate the detection of green gratings, and for these the weakening of a more red-sensitive surround yields only a weak increase in sensitivity. Note, that no sensitivity decreases are described by the present theory.

\section{Comparison with Psychophysical Results}

In many experiments, a red and a green pattern differing in a suitable spatial or movement parameter (see below) were inspected ("adaptation"). In an alternating sequence, the patterns were presented to the same part of the retina but fixation was not required. In order to reveal the aftereffect, two juxtaposed or successively appearing black-white test gratings were viewed which differed in the same spatial or movement parameter as during adaptation. Complementary hues reinforced by simultaneous or successive color contrast, respectively, appeared in the composite test pattern.

The present theory correctly describes several color aftereffects which have been observed with this technique. The varied parameter in gratings was: orientation (McCollough, 1965), spatial frequency (Breitmeyer \& Cooper, 1972; Lovegrove \& Over, 1972), direction of curvature convexity (Riggs, 1973), direction of movement (Hepler, 1968), and a scale factor in dot patterns (MacKay \& MacKay, 1975a).

The following further results can be described by the present theory:
(1) Complementary color aftereffects for colorwhite adaptation gratings (Harris \& Barkow, 1969; these authors found no aftereffect for color-gray gratings, whereas the present theory predicts a weak positively colored aftereffect).

(2) Absence of aftereffects for fixated red-green and color-gray gratings (Stromeyer \& Dawson, 1978).

(3) Complementary color aftereffects for fixated color-black and color-white gratings apparent only when, during testing, the white bars fall in the positions adapted to the colored in the former case and the white bars in the latter (Stromeyer \& Dawson, 1978).

(4) In darkness, the McCollough effect apparently persists indefinitely (MacKay \& MacKay, 1974, 1975b). The long persistence of the effect during viewing of natural scenes has been noted by many workers, beginning with McCollough (1965).

(5) The McCollough effect is not accompanied by large sensitivity decreases for the adapting stimulus (Timney, Gentry, Skowbo, \& Morant, 1974). Since sensitivity increases have apparently not been expected by these authors, the results of threshold measurements in their Figure 1 have been reevaluated. These data have been obtained by Timney et al. $1 / 2 \mathrm{~h}$ after adaptation to a red vertical and a green horizontal grating. For the present analysis, they have been pooled together for two observers, three threshold criteria, and three values for a "red" and a "green" half axis of the wavelength scale. Sensitivities after adaptation were higher than before for the following numbers of single measurements: green vertical test grating: 10 out of 18 ; red horizontal test grating: 12 out of 18; red vertical test grating: 3 out of 18 ; green horizontal test grating: $6 \frac{1 / 2}{2}$ out of 18 ; with $1 / 2$ standing for equality.

As can be seen, there is a majority of single measurements (first two cases) where the adaptation to a grating of a given color results in an increased sensitivity to a grating of the complementary color. This change, although very small, is possibly significant, particularly when compared to the predominantly lowered sensitivites to the adapting gratings themselves (bottom two rows). This may be either a residue of the large unspecific sensitivity decrease shortly after adaptation (mentioned by Timney et al., 1974) or a sign of orthogonal color opponency (i.e., a rigid link of green vertical sensitivity increase and red horizontal decrease.) A weak effect of this kind could, in principle, be described by the present theory (Section 7), when I-cell endings less active than the mean are allowed to translate their morethan-mean share of restored metabolites into a morethan-normal synaptic efficiency. But even then, during testing in an orthogonal direction after adaptation to one colored grating alone, the altered stripes in the surrounds are not well matched by the test 
grating bars. In the literature, however, there is no evidence for orthogonal color opponency (MacKay \& MacKay, 1977; Stromeyer, 1978).

\section{Comparison with Other Theories}

The present theory has features in common with the following theoretical proposals:

(1) Harris and Gibson (1968) suggest that the McCollough effect is due to the adaptation of neuronal elements which are light sensitive at two adjacent retinal loci ("dipoles"). The isolated synapse transmitting intrageniculate lateral inhibition (Figure 2b) can be considered as such an element, since it is influenced by the left optic afferent and an I-cell situated at the right with neighboring receptive fields. Deductions of orientation tuning sharpness from dipole characteristics (Stromeyer, 1978) must, however, take into account that the output of a P-cell is determined by many such dipoles. Moreover, diameters of receptive field surrounds of P-cells would have to be known.

(2) MacKay and MacKay (1973, 1974) suggest cooperative synaptic changes in the retina or LGN to be responsible for the McCollough effect. According to the present theory, the immediate effects of adaptation ("fatigue") occur in isolation, but thereafter the cell body of an I-cell establishes a metabolic interrelation between all output endings of the cell.

(3) Creutzfeldt (1973) suggests the formation of links between (cortical) cells sensitive to orientation and others to color. As in the present theory, modifications of lateral, instead of output, connections are thought to be crucial.

Apparently only one formal theory of the McCollough effect has been published so far (Montalvo, 1976): It is a learning model which describes the formation of receptive fields specific for both orientation and color, resulting from inspection of adapting patterns. The development of positive color aftereffects is circumvented by the assumption that the formation of, for example, strongly redsensitive cells leads to a depletion of red sensitivity in more achromatic cells, which then signal "greenish" upon exposure to an achromatic pattern. This theory probably can be made to describe the adaptation to a variety of patterns and contrasts. However, it predicts a positive hue shift when the McCollough effect is tested with an entirely monochromatic pattern, whereas complementary hue shifts are found experimentally (Stromeyer, 1974).

Many authors suggest that adaptation, or fatigue of cortical cells, with appropriate stimulus requirements might explain the observed effects. This proposal is difficult to reconcile with electrophysiology, since monocular, but highly specific, cortical cells seem to be rare (Hubel \& Wiesel, 1968; Poggio \& Fischer, 1977).

\section{Discussion}

Suggestions for the explanation of some further experimental results. Binocular interactions have not been considered, since available physiological and anatomical results do not permit deriving wellfounded assumptions, although Holländer and Martinez-Millán (1975) have described a backprojection from cortex to LGN, which by its layer of origin is probably binocular. When a McCollough pattern is inspected monocularly, but testing is done binocularly, the aftereffect in the adapted eye is found to be weakened, and a reverse aftereffect builds up in the unadapted eye (Mikaelian, 1975). This could be interpreted as an attempt of selfannihilation of the aftereffect by binocular hue feedback from cortex to LGN.

When, after adaptation to a moving colored grating, testing is done with a stationary grating of the same color, apparent movement is seen (Favreau, Emerson, \& Corballis, 1972; Hepler, 1968; Mayhew \& Anstis, 1972). According to the present theory, the LGN P-cells acquire a color-dependent specificity to the direction of movement, so that the velocity tuning of cortical cells becomes skewed, and cells signaling a low nonzero velocity may become more responsive to a stationary colored grating than those signaling zero velocity. Note that this is expected even in cortical cells with perfectly symmetric red and green-center LGN input.

The present theory cannot explain aftereffects involving apparent tilts related to colors (Held \& Shattuck, 1971; Lovegrove \& Over, 1973; Mikaelian, 1976). The second authors have noted that some of these effects decay rapidly, and therefore a separate mechanism might be responsible.

In Section 6, an achromatic McCollough effect consisting in an increase of grating contrast is predicted. So far, there is no clear experimental evidence for such an effect, and it could only be detected when the achromatic spatial frequency aftereffect yielding a contrast decrease (Blakemore \& Campbell, 1969) has faded away. (The latter effect differs in a number of ways from the McCollough effect, and because of strong binocular transfer, it is thought to occur in the visual cortex.) Mayhew and Anstis (1972) have described several achromatic form-contingent motion aftereffects, some of which can be described by the present theory, but the authors make no explicit statement on the long persistence of these achromatic effects.

McCollough effect as a model for memory. The present theory relates the McCollough effect in an early stage to simple fatigue (Assumption 2a), but later to the difficulty of metabolite restoration that is faced by a neuron with locally variable output (Assumption 2b). These two stages could be taken to correspond to short-term and long-term memory, the former entailing the latter. It is understood that insufficiently restored metabolites must not necessarily represent the material substrate of memory, but these might trigger other macromolecular events. 
This model is analogous to passive memory, since the greater part of the stored information, namely the pattern, must be furnished, in order to elicit the recall of the small amount of information contained in the hue.

Possible alternatives. In the construction of this theory, a number of assumptions have been made. Only one of them, namely the choice of the type of connectivity (Figure 1) and the resulting autostroboscopic impressing, is essential to the system and could not be replaced without changing the basic idea. However, the following modifications can be considered:

(1) Instead of a total inability to restore quantitatively the metabolites consumed locally (Assumption 2b), it is sufficient, and more realistic, to assume that the cell is nearly, but not perfectly, able to meet the local demands. Similarly, a more realistic intensity-response curve can be chosen for the gating function. It must only exceed the I-cell's intensity-response function at low intensities, and be saturated at lower intensities than the latter. Moreover, the action of the facilitatory synapse need not range from maximum ("open") to zero ("closed"), but only down to a positive minimum so that the intrageniculate surround could well have a steadily functioning component.

(2) The above types of intensity-response functions for the center gate and the I-cell response may be interchanged, and at the same time strengthening, instead of weakening, of lateral connections be introduced. This has one advantage for the present theory: The results of the foregoing sections are described as well, but, in addition, the appearance of complementary color fringes after adaptation to prisms (Gibson, 1933) can be more easily understood. In the present formulation, on-center and off-center cells yield results which are opposed to each other so that the net result unduly depends on parameter choices. Therefore, from this example alone it could not be decided which version was more appropriate. The interchanged version has been refuted, since the surround mechanism would be somewhat unconventional, showing well-graded responses only as a function of the center illumination.

(3) Instead of perfectly sustained responses of all cells involved, transient components can be introduced. Larger, but qualitatively similar, effects for moving patterns can then be obtained, and the ineffectiveness of retinally stabilized adaptation patterns (Piggins \& Leppmann, 1973) can be understood.

(4) A cell playing the role of the P-cell could itself activate the gating mechanism via some unspecified backward action, thereby obviating the need for a separate facilitatory axon collateral. Any cell, for example in the cortex, with inputs corresponding to center excitation and color-opponent surround inhibition could then show the phenomena described by the present theory. However, as long as corresponding physiological evidence is lacking, it seems preferable to identify the proposed mechanism at least with a suggestive anatomical structure such as the one found in the monkey LGN.

Conclusion. It has been demonstrated that many variants of the McCollough effect can be explained by only a few types of color-opponent cells with a concentric center-surround organization. In the rhesus monkey, these types are known to be most abundant at the LGN dorsal layer level, and therefore an arbitrary attribution of McCollough effect properties to particular subsets of cells is unnecessary. Moreover, the alteration of synaptic efficiency due to activity transmitted, and the metabolic machinery responsible for efficiency maintenance, can be assumed to be identical for all cells and synapses contained in the considered network, and yet, different types of synapses show differences in persistence of alterations. These differences are related to the network connectivity, and the synapses showing the longpersisting changes are just those which are critically involved in the adaptation to the stimuli which are known to produce a McCollough effect. The theory permits an understanding of several, so far unexplained, observations, namely adaptation results with various degrees of luminance contrast and the absence of large threshold increases associated with the aftereffects. Moreover, the need to decide whether curvature-contingent aftereffects can be explained by adaptation to elements of tilted straight-line elements or whether genuine curvature is involved (for seven references see Stromeyer, 1978) is obviated. Even if the present theory should prove to give an incorrect description of reality, it clearly shows that, from psychophysics alone, one cannot conclude that the McCollough effect furnishes evidence for the existence of stimulus-specific channels, or cells, which adapt. Rather, it seems that in several respects the aftereffects are just as specific as an amorphous neural substrate has been made by adaptation.

\section{REFERENCES}

Blakemore, C., \& CAMPBELL, F. W. On the existence of neurons in the human visual system selectively sensitive to the orientation and size of retinal images. Journal of Physiology, 1969, 203, 237-260.

Breitmeyer, B. G., \& CoOper, L. A. Frequency-specific color adaptation in the human visual system. Perception \& Psychophysics, 1972, 11, 95-96.

Cleland, B. G., \& Dubin, M. W. The intrinsic connectivity of the LGN of the cat. Experimental Brain Research Supplement, $1976,1,493-496$.

Colonnier, M., \& GULlery, R. W. Synaptic organization in the lateral geniculate body of the monkey. Zeitschrift für Zellforschung, 1964, 62, 333-355.

Creutzfeld T, O. D. Some neurophysiological considerations concerning "memory." In H. P. Zippel (Ed.), Memory and transfer of information. New York: Plenum, 1973.

DeMonasterio, F. M., \& Gouras, P. Functional properties of ganglion cells of the rhesus monkey. Journal of Physiology, 1975, $251,167-196$.

Favreau, O. E., Emerson, V. F., \& Corballis, M. C. Motion perception: A color-contingent aftereffect. Science, 1972, 176, 78.79.

Gibson, J. J. Adaptation, after-effect and contrast in the perception of curved lines. Journal of Experimental Psychology, 1933, 16, 1-31.

GouRAs, P. Identification of cone mechanisms in monkey ganglion cells. Journal of Physiology, 1968, 199, 533-547.

GraY, E. G. Electron microscopy of excitatory and inhibitory synapses: A brief review. Progress in Brain Research, 1969, 31, 141-155.

Hámori, J., Pasik, T., Pasik, P., \& Szentágothar, J. Triadic synaptic arrangements and their possible significance in the lateral geniculate nucleus of the monkey. Brain Research, 1974, 80, 379-393.

HARrIs, C. S., \& Barkow, B. Color/white grids produce weaker orientation-specific aftereffects than do color/black grids. Psychonomic Science, 1969, 17, 123.

HARRIS, C. S., \& Gibson, A. R. Is orientation-specific color adap- 
tation in human vision due to edge detectors, afterimages or "dipoles"? Science, 1968, 162, 1506-1507.

Helo, R., \& Shattuck, S. R. Colot- and edge-sensitive channels in the human visual system: Tuning for orientation. Science, 1971,. 174, 314-316.

HePLER, N. K. Color: A motion contingent aftereffect. Science, $1968,162,376-377$.

Holländer, H., \& MARTinez-Millán, L. Autoradiographic evidence for a topographically organized projection from the striate cortex to the lateral geniculate nucleus in the rhesus monkey (Macaca mulatta). Brain Research, 1975, 100, 407-411.

Hubel, D. H., \& Wiesel, T. N. Receptive fields and functional architecture of monkey striate cortex. Journal of Physiology, $1968,195,215-243$.

Jameson, D., \& HuRvich, L. M. Opponent-response functions related to measured cone photopigments. Journal of the Optical Society of America, 1968, 58, 429-430.

KRÜGER, J. Stimulus dependent colour specificity of monkey lateral geniculate neurones. Experimental Brain Research, 1977, 30, 297.311.

LEVAY, S. On the neurons and synapses of the lateral geniculate nucleus of the monkey and the effects of eye enucleation. Zeitschrift fur Zellforschung, 1971, 113, 396-419.

LOVEgrove, W. J., \& Over, R. Color adaptation of spatial frequency detectors in the human visual system. Science, 1972, 176, 541.543.

LOVEgrove, W. J., \& OVER, R. Color selectivity in orientation masking and aftereffect. Vision Research, 1973, 13, 895-901.

MACKAY, D. M., \& MACKAY, V. Orientation-sensitive aftereffects of dichoptically presented colour and form. Nature (London), 1973, 242, 477-479.

MacKay, D. M., \& MacKay, V. The time course of the McCollough effect and its physiological implications. Journal of Physiology, 1974, 237, 38-39.

MACKAY, D. M., \& MACKaY, V. Dichoptic induction of McCollough-type effects. Quarterly Journal of Experimental Psychology, 1975, 27, 225-233. (a)

MacKaY, D. M., \& MacKay, V. What causes decay of patterncontingent chromatic aftereffects? Vision Research, 1975, 15, 462-464. (b)

MACKAY, D. M., \& MACKAY, V. Multiple orientation-contingent chromatic aftereffects. Quarterly Journal of Experimental Psychology, 1977, 29, 181-202.

Mayhew, J. E. W., \& Anstis, S. M. Movement aftereffects contingent on color, intensity, and pattern. Perception \& Psychophysics, 1972, 12, 77-85.
McCollough, C. Color adaptation of edge detectors in the human visual system. Science, $1965,149,1115-1116$.

MikaELIAN, H. H. Interocular generalization of orientation specific color aftereffects. Vision Research, 1975, 15, 661-663.

Mikaelian, H. H. Plasticity of orientation specific chromatic aftereffects. Vision Research, 1976, 16, 459-462.

Montalvo, F. S. A neural network model of the McCollough effect. Biological Cybernetics, 1976, 25, 49-56.

Pecci-SaAvedra, J., \& Vaccarezza, O. L. Synaptic organization of the glomerular complexes in the lateral geniculate body of cebus monkey. Brain Research, 1968, 8, 389-393.

Piggins, D. J., \& LeppManN, P. K. Role of retinal image motion in evoking the McCollough effect. Nature New Biology, 1973, 245, 255-256.

Poggio, G. F., \& Fischer, B. Binocular interaction and depth sensitivity in striate and prestriate cortex of behaving rhesus monkey. Journal of Neurophysiology, 1977, 40, 1392-1405.

Riggs, L. A. Curvature as a feature of pattern vision. Science, 1973, 181, 1070-1072.

Skowbo, D., Timney, B. N., Gentry, T. A., \& Morant, R. B. McCollough effects: Experimental findings and theoretical accounts. Psychological Bulletin, 1975, 82, 497-510.

STROME YER, C. F., III. Form-specific colour aftereffects observed in scotopic illumination. Nature (London), 1974, 250, 266-268.

Strome Yer, C. F., III. Form-color aftereffects in human vision. In R. Held, H. W. Leibowitz, \& H. L. Teuber (Eds.), Handbook of sensory physiology (Vol. 8) Perception. Berlin: Springer-Verlag, 1978.

STrome Yer, C. F., III, \& DAwson, B. M. Form-color aftereffects: Selectivity to local luminance contrast. Perception, 1978, 7, 407-415.

SzentáGothai, J. Neuronal and synaptic architecture of the lateral geniculate body. In R. Jung (Ed.), Handbook of sensory physiology (Vol. VII/3B) Central processing of visual information. Berlin: Springer-Verlag, 1973.

Timney, B. N., Gentry, T. A., Skowbo, D., \& Morant, R. B. Chromatic grating thresholds and the McCollough effect. Vision Research, 1974, 14, 1033-1035.

Wiesel, T. N., \& Hubel, D. H. Spatial and chromatic interactions in the lateral geniculate body of the rhesus monkey. Journal of Neurophysiology, 1966, 29, 1115-1156.

(Received for publication June 21, 1978; revision accepted August 26, 1978.) 